\title{
Study on a wide field-of-view Cherenkov telescope with large dimensional refractive lens for high energy Cosmic Rays detection
}

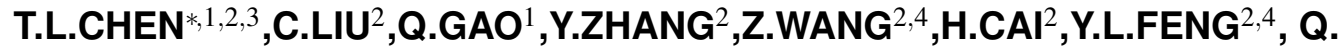 \\ WANG $^{2}$,Y.Q.GUO ${ }^{2}$,H.B.HU ${ }^{2}$, Y.SHI $^{3}$, DANZENGLUOBU ${ }^{1}$, M.Y.LIU ${ }^{1}$, Q. B. GOU ${ }^{2}$ \\ ${ }^{1}$ The Key Laboratory of Cosmic Rays (Tibet University), Ministry of Education, Lhasa 850000, \\ Tibet, China \\ ${ }^{2}$ Key Laboratory of Particle Astrophysics, Institute of High Energy Physics, Chinese Academy of \\ Sciences, Beijing 100049, China \\ ${ }^{3}$ School of Physics and Technology, Wuhan University, Wuhan 430072, Hubei, China \\ ${ }^{4}$ University of Chinese Academy of Sciences, Beijing 100049, China \\ E-mail: fbc1980@163.com (Q.GAO), zhangyi@ihep.ac.cn(Y.ZHANG) ${ }^{\dagger}$
}

\begin{abstract}
We are developing a wide field-of-view Cherenkov telescope with large dimensional refractive lens for sub-100 GeV to $\mathrm{TeV}$ gamma ray astronomy. A prototype of the refractive telescope with a camera composing of 48 PMTs and the front-end electronics is designed, assembled, and tested to demonstrate the feasibility of the concept. In this work, we report some preliminary results on the detection of high energy Cosmic Rays (CRs) using the prototype telescope which was operated in coincidence with a scintillator detector array at the Yangbajing Observatory in Tibet.The prototype telescope can achieve a $15^{\circ} \times 11^{\circ} \mathrm{FoV}$ and a $\sim 0.8^{\circ}$ angular resolution which is suitable to take certain Cherenkov images for air showers.
\end{abstract}

35th International Cosmic Ray Conference - ICRC2017

10-20 July, 2017

Bexco, Busan, Korea

\footnotetext{
${ }^{*}$ Speaker.

$\dagger$ This work was supported by the Research Project of Chinese Ministry of Education (No. 213036A), the Grants from the National Natural Science Foundation of China (Nos. 11563007,11135010, 11105156, 11405180, 11463004, 11375209, 11635011, 11647311 and 11663006), the 973 Program of China (No. 2013CB837000) and Youth Innovation Promotion Association, Chinese Academy of Sciences.The speaker T.L.CHEN is also supported by Talents Program for Mount Qomolangma Scholars of Tibet University.
} 


\section{Introduction}

The energy region around sub- $100 \mathrm{GeV}$ is a crucial bandwidth in $\gamma$-ray astronomy, which is the connection between the space and ground-based observation. The observation in this energy range could give important messages about $\gamma$-ray Bursts (GRBs), Active Galactic Nucleus (AGNs), and Extragalactic Background Light (EBL) by investigating these sources, and hence to study the galaxy formation and the evolution of the early universe. The analysis of the light curve of the transient source is one of the most sensitive methods to constrain the Lorentz Invariance Violation (LIV).

The low sensitivity at sub-100 GeV is the weakness of the current Cosmic Rays (CRs) detecting techniques. The Fermi-LAT is performing the deepest survey in space of the $\gamma$-ray sky from $20 \mathrm{MeV}$ up to $100 \mathrm{GeV}$, but with limited sensitivity above $10 \mathrm{GeV}$ due to its limited detector size $\left(\sim 1 \mathrm{~m}^{2}\right)$. On the other hand, Imaging Atmospheric Cherenkov Telescopes (IACTs), such as the MAGIC, H.E.S.S.,VERITAS or CTA arrays, have large efficient areas of more than $10^{5} \mathrm{~m}^{2}$, but with limited a FoV on the order of tens of square degree and low duty cycle. Though having a much larger FoV of $1 \mathrm{Sr}$ and high duty cycle in addition to the comparable collection area of IACTs, air-shower array experiments (such as Tibet AS $\gamma$, ARGO-YBJ, HAWC and future LHAASO) have a lower sensitivity at sub-100 GeV mainly because of the low detecting efficiency and poor ability in particle identification.

Larger FoV and lower energy threshold are the two most important goals in detecting high energy GRBs at sub- $100 \mathrm{GeV}$ by ground-based $\gamma$-ray experiments. Allowing simultaneous coverage of a significant fraction of the sky is recognised as a high priority goal in the development of the IACT technique. MACHETE have a camera with a FOV of $5 \times 60$ square degrees which can survey half of the Very High Energy (VHE) $\gamma$-ray sky in a year have been proposed [10]. Ultra high energy CRs detectors using the fluorescence technique with a very large FoV on the order of steradian are already operational or under design [3].Very large FoV IACT technique based on refractive optics are proposed,such as JEM-EUSO [4], GAW [5] and FAST [6].

Because of the high transmittance of purified water for ultra-violate photos, we proposed that a refractive water-lens telescope with acrylic shell as light collector for observing Cherenkov light induced by CRs and high energy $\gamma$-ray, especially aiming for detecting the high energy emission of GRBs. Ideal water-lens should be in a hemispherical shape, which allows a very large FoV and can achieve a uniform image quality on the curved focus surface for all incident angle incident light. As a pathfinder, we designed and manufactured a water-lens prototype with thin spherical cap. We successfully observed Cherenkov signal induced by high energy CRs in coincidence with a scintillator Extensive Air Shower (EAS) array in Yangbajing Observatory.

\section{Experiment}

In order to test the feasibility of the new approach for measuring the atmospheric Cherenkov light induced by $\gamma$-ray, firstly, we use the above lens to collect the atmospheric Cherenkov light induced by VHE CRs. Meanwhile, an EAS array with 75 scintillator detectors has been used as a coincident measurement with the prototype system. The coincident measurement system is shown in Figure1, the red solid squares are the scintillation counters and the solid blue cycle indicate the 
water-lens telescope. The telescope is located at the southeast corner, about $80 \mathrm{~m}$ away from the center of the array. For the CR events with cores locating inside the array, the telescope would be within the light pool of those events.

The experiment has been conducted and successfully run at the Yangbajing Observatory (4300 $\mathrm{m}$ above sea level, $90.522^{\circ} \mathrm{E}, 30.102^{\circ} \mathrm{N}, 606 \mathrm{~g} / \mathrm{cm}^{2}$ ) in Tibet, China. The Yangbajing Observatory mainly hosts two EAS array experiments, Tibet AS $\gamma$ experiment and ARGO-YBJ experiment, and the LHAASO/WFCTA [7] prototype telescopes have been also tested at the site of Yangbajing Observtory.

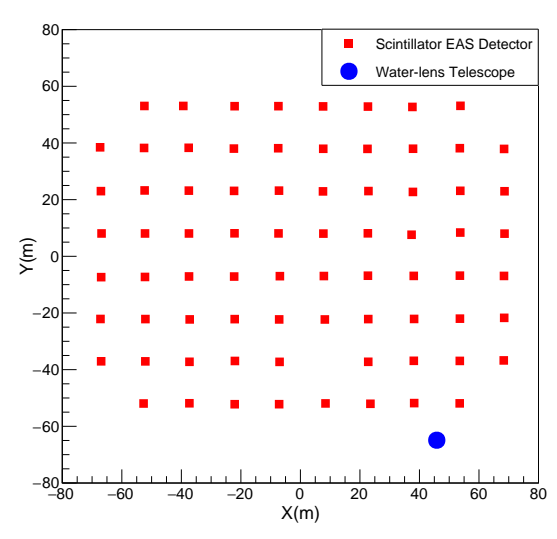

Figure 1: Layout of the coincidence expeiment.

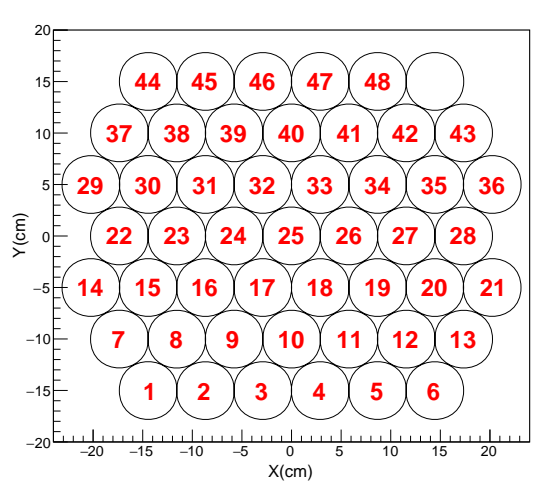

Figure 2: Configuration of camera.

\subsection{The water-lens telescope}

The prototype lens is a thin spherical cap shell filled with purified water. The surface accuracy of lens is $\pm 1 \mathrm{~mm}$ assessed by industrial Computerized Tomography (CT). The main parameters [2] of the water-lens prototype are described in Table1.

Table 1: Parameters of the water-lens

\begin{tabular}{ll}
\hline Lens diameter & $0.9 \mathrm{~m}$ \\
$F$-number & 1.8 \\
Spot size $(420-540 \mathrm{~nm})$ & $\leq 2 \mathrm{~cm}$ \\
Transmittance $(420-540 \mathrm{~nm})$ & $86 \%$ \\
Field of View & $15^{\circ} \times 11^{\circ}$ \\
Mass & $100 \mathrm{~kg}$ \\
Surface accuracy & $\pm 1 \mathrm{~mm}$ \\
\hline
\end{tabular}

In the experiment, the Photomultiplier (PMT) of Hamamatsu R7725 was selected as the photon detectors. R7725 is a head-on type PMT with bialkali photocathode, the diameter of which is $51 \mathrm{~mm}$ ( 2 inch). The spectral response is $300-650 \mathrm{~nm}$ with $420 \mathrm{~nm}$ at maximum. The camera is 
formed with 48 PMTs, the configuration of which is illustrated in Figure2. The Front-end electronics (FEE) and Data acquisition system (DAQ) of the prototype were mainly built by commercial modules. The system is schematically shown in Figure3. The PMT signal from the anode is split to two signals. One is sent to the Switched Capacitor Digitizer (SCD) (CAEN DT5742B, 16+1 Channel 12-bit $5 \mathrm{GS} / \mathrm{s}$ ). The other is sent to the low threshold discriminator (CAEN N845). The discriminator generates a logical signal with its amplitude proportional to the input signal multiplicity and output to the quad linear Fan-in/Fan-out (CAEN N625). The Fan-in/Fan-out sums the logical signals from three discriminators and output to the second discriminator. In case the signal is over the threshold of the second discriminator, the trigger is generated and sent to SCD and the FEE. When the trigger stops holding phase, the analog memory buffer of SCD is frozen. Then in the SCD, the cell content is made available to the 12 bit ADC for the digital conversion and all the waveforms of the signals are recorded. In the experiment, the sampling frequency is programmed at $2.5 \mathrm{GHz}$. A VETO was set in the system using the Dual Timer Model (CAEN N93B) due to the dead time of the SCD. The Qual Scaler (CAEN N1145) is used as well for the real-time monitor on the event rate. The threshold for each channel is set at the amplitude of the signal in case 10 photons are simultaneously obtained at the same PMT. When the amplitude of one PMT was above the threshold, the trigger signal was sent to all the SCDs. All the signals of 48 channels are recorded in a time window of $400 \mathrm{~ns}$. This trigger logic is optimized to reduce the fluctuation of the NSB and lower the threshold of the CRs.

By the geometrical optics calculation,the FoV of the telescope is $15^{\circ} \times 11^{\circ}$ and the FoV of each PMT is $1.73^{\circ} \times 1.73^{\circ}$ averagely.

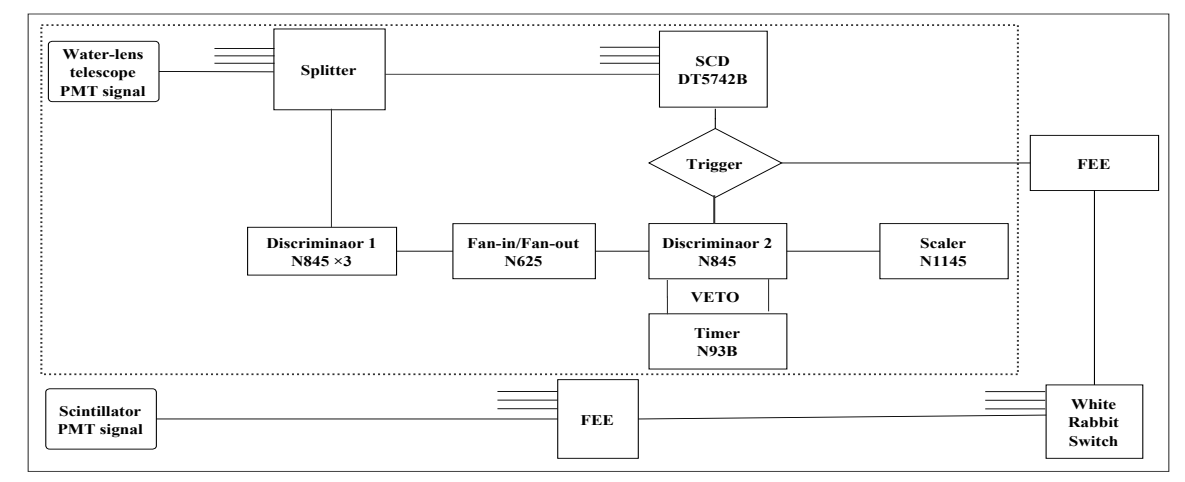

Figure 3: Schematic view of the DAQ system.

\subsection{Scintillator EAS array}

The scintillator array consist of 75 scintillation counters forming a matrix of $15 \mathrm{~m}$ span occupying an area of $18,000 \mathrm{~m}^{2}$. The most probable proton energy of the CRs observed is of $20 \mathrm{TeV}$. Each counter has a plastic scintillation plate (EJ-200) of $0.5 \mathrm{~m}^{2}$ in area and $2 \mathrm{~cm}$ in thickness and is equipped with a photomultiplier tube (Hamamatsu R7725). An upside-down pyramidal style is used as light guider. The DAQ system adopt the EDs scheme of the LHAASO KM2A array [8] and 
the trigger signal of the water-lens telescope is sent to the ED FEE as one channel and synchronized by the White Rabbit Switch.

Signals from both the anode and the ninth dynode of the photomultiplier tube (PMT) are read out and thus a dynamic range is obtained between 1 and 500 MIPs. The angular resolution of the array is $0.9^{\circ}$ at the energy of $20 \mathrm{TeV}$, as estimated by a full Monte Carlo (MC) simulation. The event rate is about $26 \mathrm{~Hz}$.

The event selection was done by imposing the following four criteria on the reconstructed data: Each shower event should have four or more detectors fired (i.e. $n c h$ ) and recording 1.25 or more particles each; The estimated shower center location should be inside the array; The sum of the number of particles per $m^{2}$ (i.e. $\Sigma \rho$ ) detected in each detector should be larger than 15; The zenith angle (i.e. $\theta$ ) of the incident direction should be less than 30 . After applying these cuts and a data quality cut, about $40 \%$ of the shower events were selected in analysis.

\section{Results}

\subsection{PMT pointing and bright star}

When a bright star enters the FoV of the telescope, the trigger rate will increased. As the camera has 48 pixel, the track of the star can be obtained. For a certain pixel, the time interval influenced by one bright star is related to the imaging quality of the lens and the size of the pixel.

Figure 4 shown the normalized trigger rate of the $21^{\text {th }}$ PMT within 4 hours in three clear moonless night. The peaks due to stars are clearly seen, by using TD 1 Catalog ${ }^{1}$ (This catalog was also used by LHAASO/WFCTA [9]) of Stellar UV Fluxes, we knew the highest peak is caused by HD358 (Alpha Andromedae). The black, red, blue line indicate three continuous moonless nights, $3^{\text {rd }}, 4^{\text {th }}, 5^{\text {th }}$ November 2016. We take HD358 as an example. In case the star reaches the peak, the direction of the star indicates the pointing direction of the corresponding PMT. According to the peak time and the direction of the known star (R.A., Dec.), we can calculate the pointing direction of the PMT. We define the pointing direction using $\theta$ (zenith angle), $\phi$ (azimuth angle) and expressing as $(\theta, \phi)$. The pointing direction of the $21^{\text {th }}$ PMT is $\left(4.6 \pm 0.7^{\circ}, 258.5 \pm 2.3^{\circ}\right)$ using bright star information, and which is within the expected point range $\left(\theta \in\left(3.3^{\circ}, 4.9^{\circ}\right), \phi \in\left(236.3^{\circ}, 258.9^{\circ}\right)\right)$ decided by the place and diameter of the PMT. On the basis of the time that the star passed through the telescope, the FoV of the telescope in the E-W direction could be inferred, which is $15.4^{\circ}$ and basically close to the geometry calculated result $\left(15.3^{\circ}\right)$.

\subsection{Off-line coincidence with the scintillator EAS array}

From $3^{\text {rd }}$ to $6^{\text {th }}$ November 2016, the experiment has been run for 4 nights ( 31.5 hours) stably and under the same conditions. $1.79 \times 10^{7}$ events has been collected and the trigger rate is $N_{1}=157.7$ Hz. At the same time, $2.95 \times 10^{6}$ events have pass the criteria of the scintillator EAS array with the event rate of $N_{2}=26 \mathrm{~Hz}$. The coincident time window is set at $400 \mathrm{~ns}$. In this work, the number of coincident event is $5.18 \times 10^{4}$ and the coincident event rate is $N=0.46 \mathrm{~Hz}$. The accidental coincident

\footnotetext{
${ }^{1}$ https://heasarc.gsfc.nasa.gov/w3browse/all/tdl.html
} 


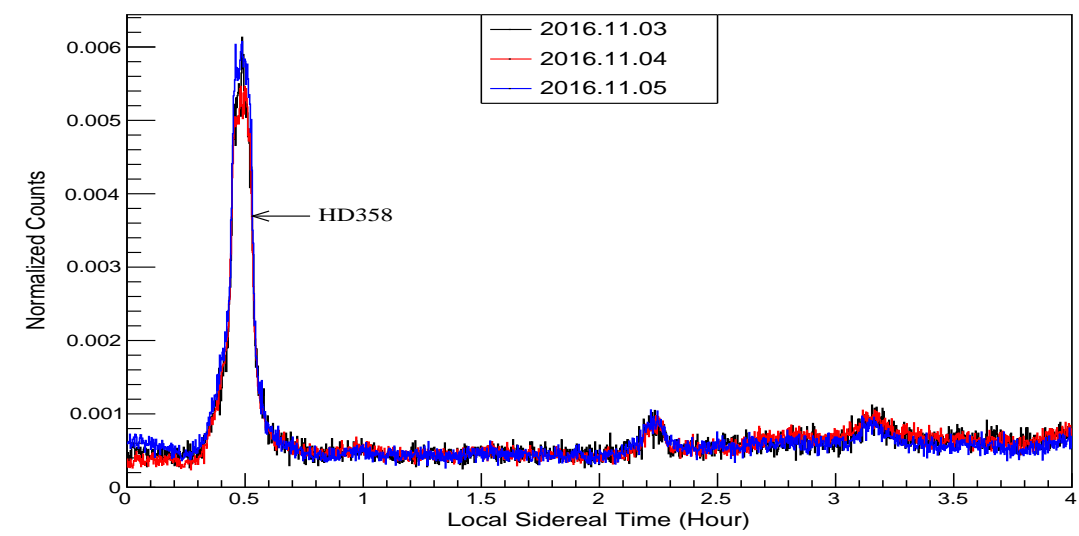

Figure 4: Normalized event rate of $21^{\text {th }}$ PMT.

rate was $R=2 N_{1} N_{2} \tau=0.003 \mathrm{~Hz}$. Therefore the accidental coincidence rate can be ignored, which indicates that the coincident events are real Cherenkov light induced by CRs.

\subsection{Reconstruction of arrival direction}

In this work, a rough reconstruction method is employed in the analysis. The pointing direction of individual shower is reconstructed using geometrical optics based on centroid method. Figure 5 presents a typical CRs event obtained by the water-lens telescope with direction $\left(2.5^{\circ}\right.$, $103^{\circ}$ ). The main parameters of this event measured by the EAS array are as follows: $\sum \rho=87.8$ (77.6 TeV), direction $\left(2.5^{\circ}, 91.0^{\circ}\right)$. The shower core $(X=30.2 \mathrm{~m}, Y=1.6 \mathrm{~m})$ of this event is a $68 \mathrm{~m}$ distance to water-lens telescope $(X=45.8 \mathrm{~m}, Y=-65 \mathrm{~m})$. The result implies the water-lens telescope has capability in atmospheric Cherenkov imaging. Figure6 displayed a typical Cherenkov light recorded by $25_{\max }^{t h}$ PMT ( $i_{\max }^{\text {th }}$ represented the PMT with maximum amplitude). The full width at half maximum (FWHM) was $4.5 \mathrm{~ns}$ and the charge is about 47 photoelectrons (p.e) of the signal . Figure7 ( $a$ ) and $(b)$ demonstrated the pulse shapes of the Cherenkov signal in individual shower

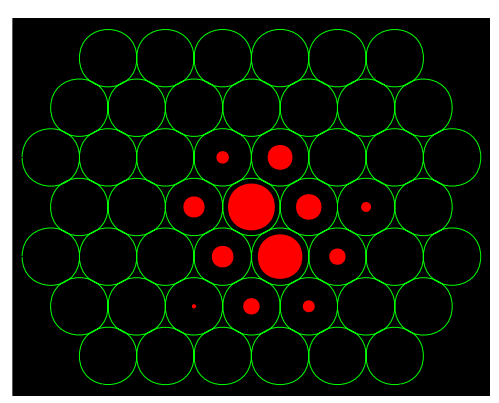

Figure 5: Air shower event.

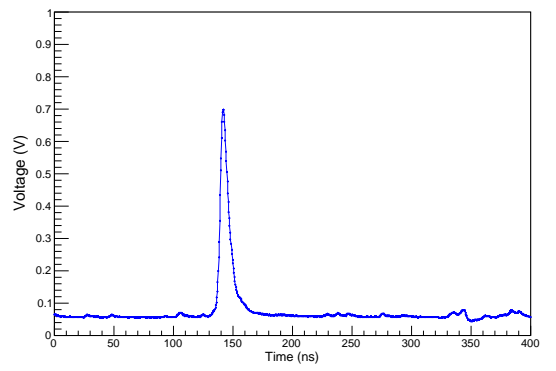

Figure 6: Cherenkov signal with NSB.

from two directions. The channel with maximum charge is $25_{\max }^{\text {th }}$. Figure7 (a) shown one direction pulse profiles (from outer to inner, $2^{\text {nd }}, 9^{\text {th }}, 17^{\text {th }}, 25^{\text {th }}, 33^{\text {th }}, 41^{\text {th }}, 48^{\text {th }}$ channel respectively, channel 
identifier see Figure2) and Figure7 (b) shown another direction pulse profiles (from outer to inner, channel No $5^{\text {th }}, 18^{\text {th }}, 25^{\text {th }}, 32^{\text {th }}, 39^{\text {th }}, 45^{\text {th }}$, respectively).

(a)

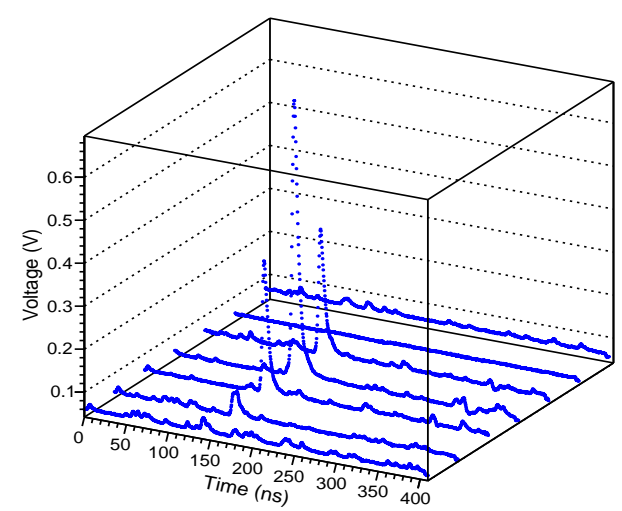

(b)

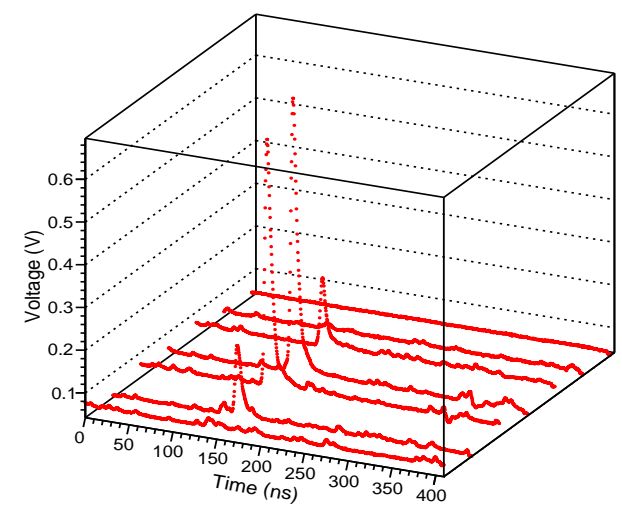

Figure 7: The Cherenkov pulse shape detected by water-lens telescope.

\subsection{Estimation of angular resolution}

The angular resolution and the pointing direction of the water-lens telescope could be estimated by the coincident events. The distribution of each $i_{\max }^{\text {th }}$ PMT events can be well described by a Gaussian distribution. The pointing direction of this PMT can also be deduced by the mean value of the Gaussian distribution. The deviation is caused both by the angular resolution of the prototype and the EAS array. Figure8 presents the direction distribution reconstructed by the EAS array in E-W and N-S direction in case the $i_{\text {max }}^{\text {th }}$ is the $21^{\text {th }}$ PMT. Here, the point direction of the $21^{\text {th }}$ PMT is $\left(4.4^{\circ}, 248.8^{\circ}\right)$, consistent with that obtained by the bright star calibration $\left(4.6 \pm 0.7^{\circ}\right.$, $\left.258.5 \pm 2.3^{\circ}\right)$. The deviation of the distribution is $1.36 \pm 0.13^{\circ}$ and $1.34 \pm 0.12^{\circ}$ in E-W and N-S direction, respectively. In this case, the resolution of the water-lens telescope estimated as $\sim 0.8^{\circ}$, consistent with the expectation.

\section{Summary and Discussion}

To develop a ground-based IACT technique allowing simultaneous coverage of significant fraction of the sky is recognised as a high priority issue. This would be very important for independent detection of solitary VHE $\gamma$-ray events, e.g. few hundreds or even few tens of $\mathrm{GeV}$ emission of GRBs. A very large Fov IACT based on refractive optics located in high elevation site is expected to achieve this goal. Instead of using Fresnel lenses, we propose water-lens as Cherenkov light collector. As a R\&D prototype, we use a $0.9 \mathrm{~m}$ diameter water-lens to verify our proposition. The Cherenkov light induced by CRs has been successfully observed in coincidence with a scintillator EAS array in Yangbajing Observatory. Using the coincidence measurement and the bright star calibration in the $\mathrm{FoV}$, the prototype telescope can achieve a $15^{\circ} \times 11^{\circ} \mathrm{FoV}$ and a $\sim 0.8^{\circ}$ angular resolution which is suitable to take certain Cherenkov images for air showers. 
(a)

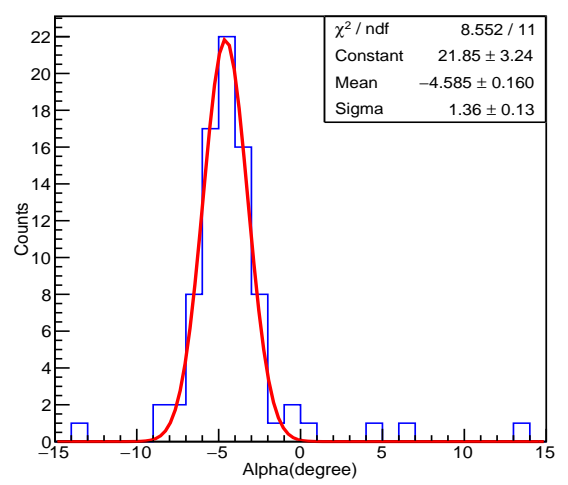

(b)

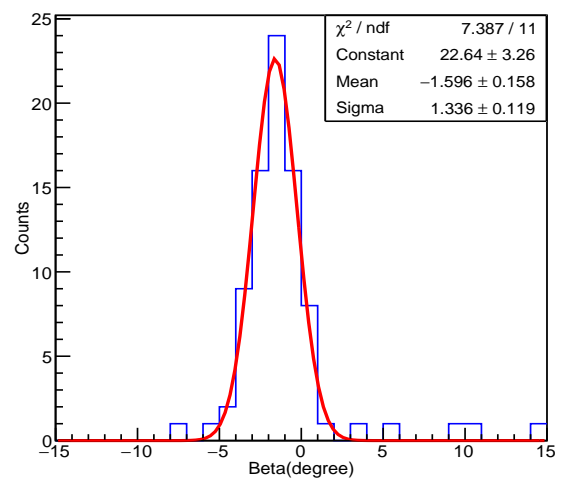

Figure 8: The angular resolution of $21^{\text {th }}$ PMT in E-W and N-S direction.

Larger dimensional spherical water-lens and array located at very high altitude observatory should have potential importance for the future $\gamma$-ray astronomy. The technology in manufacturing the lens, the mega-pixel/nanosecond/high-quantum-efficiency camera and huge data handling capability will be developed in the future.

\section{References}

[1] S. Z. Chen, T. di Girolamo, H. H.He, et.al, Prospects for Gamma Ray Bursts detection with LHAASO, in proceedings of $34^{\text {th }}$ ICRC, POS ( ICRC2015) 728 (2015).

[2] T. L. Chen, Z. Wang, Q. Gao, et.al., Study on a prototype of the large dimensional refractive lens for the future large field-of-view IACT, in proceedings of 34 th ICRC, POS ( ICRC2015) 1002 (2015).

[3] B. Bartoli, P. Bernardini, X. J. Bi, et al., Energy spectrum of cosmic protons and helium nuclei by a hybrid measurement at 4300 m a.s.l., Chinese Physics C 38 (2014), 045001.

[4] M.Ricci, The JEM-EUSO Program, Journal of Physics Conference Series 718(2016) 052034.

[5] G. Cusumano, G. Agnetta, P.Assis,, et al., Characteristics and Performance of the GAW Experiment for a Large Field of View Cerenkov Gamma-ray Telescope., Chinese Journal of Astronomy and Astrophysics Supplement 6 (2016), 369.

[6] T. Fujii, M. Malacari, M. Bertaina, et.al, Detection of ultra-high energy cosmic ray showers with a single-pixel fluorescence telescope, Astroparticle Physics 74 (2016), 64.

[7] S. S. Zhang, Y. X. Bai, Z. Cao, et.al, Properties and performance of two wide field of view Cherenkov/fluorescence telescope array prototypes, Nuclear Instruments and Methods in Physics Research A 629 (2011), 57.

[8] X. Liu, J.-F. Chang, Z. Wang, et.al, Prototype of readout electronics for the LHAASO KM2A electromagnetic particle detectors, Chinese Physics C 40 (2016), 076101

[9] L.-L. Ma, Y.-X. Bai, Z. Cao, et.al, Geometry and optics calibration of WFCTA prototype telescopes using star light, Chinese Physics C 35(2011), 478

[10] J. Cortina,R. López-Coto, A.Moralejo, MACHETE: A transit imaging atmospheric Cherenkov telescope to survey half of the very high energy $\gamma$-ray sky, AstroparticlePhysics 72(2016), 46 